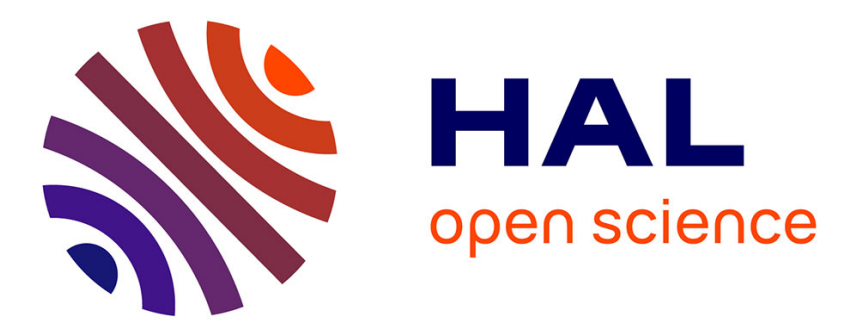

\title{
Quand Jugurta rencontre le Petit Prince. Regards rétrospectifs sur une bulle scientifique (Jugurta) \\ Philippe Gervais-Lambony
}

\section{To cite this version:}

Philippe Gervais-Lambony. Quand Jugurta rencontre le Petit Prince. Regards rétrospectifs sur une bulle scientifique (Jugurta). Revue Tiers Monde, 2016, Les Suds Aujourd'hui: retour critique sur les problématiques et les méthodologies de quelques programmes de recherche, Hors S2rie, pp.43-67. 10.3917/rtm.hs02.0043 . hal-01640550

\section{HAL Id: hal-01640550 \\ https://hal.parisnanterre.fr/hal-01640550}

Submitted on 18 Oct 2019

HAL is a multi-disciplinary open access archive for the deposit and dissemination of scientific research documents, whether they are published or not. The documents may come from teaching and research institutions in France or abroad, or from public or private research centers.
L'archive ouverte pluridisciplinaire $\mathbf{H A L}$, est destinée au dépôt et à la diffusion de documents scientifiques de niveau recherche, publiés ou non, émanant des établissements d'enseignement et de recherche français ou étrangers, des laboratoires publics ou privés. 


\section{Quand Jugurta rencontre le Petit Prince. Regards rétrospectifs sur une bulle scientifique (Jugurta)}

\section{Philippe Gervais-Lambony}

\section{MOTS-CLÉS}

Recherche, méthodologie, justice spaciale, Afrique, décentrement.

\section{RÉSUMÉ}

En référence à Jugurtha, roi de Numidie, considéré comme un dangereux barbare à Rome et un héros en Afrique, le sigle du projet visait à rappeler que, parmi ses objectifs scientifiques, figurait l'adoption d'un point de vue sur les questions urbaines depuis les Suds, en particulier depuis le continent africain. La réflexion d'ordre général est donc caractérisée par le fait qu'elle s'appuie sur des terrains de recherche habituellement abordés principalement sous l'angle du " développement ». Or, il s'agit ici de revendiquer le droit entier de ces terrains à alimenter des débats théoriques généraux; en particulier le concept de " justice spatiale ", c'est-à-dire l'approche spatiale de la justice sociale entendue dans ses différentes dimensions, tant de distribution équitable que de "reconnaissance ", a été au cœur de la réflexion d'un groupe de 30 chercheurs, divers par leurs terrains mais aussi par leurs thématiques habituelles, leur pays d'origine et leur génération.

Dans une période tendue pour l'université et la recherche en France, marquée par des évolutions structurelles fortes, des rupture temporelles et des débats sur les conditions d'exercice du métier d'enseignantchercheur, un programme de recherche financé, comme Jugurta, se présente comme une "bulle " sortie du temps ordinaire, une bulle dont on s'efforcera d'explorer et de décrire les conditions de constitution 
d'abord, le mode de fonctionnement interne ensuite, les voies pour en prolonger l'existence sous d'autres formes enfin. Cette réflexion porte donc simultanément sur les manières de " chercher " (en réseau et lors d'une parenthèse temporelle) et ses effets sur ce qui est " trouvé ", sur les méthodes et les temporalités de la recherche, et leurs conséquences sur ce qu'il est convenu d'appeler des "résultats". 


\section{Introduction}

«Lorsque j'avais six ans j'ai vu, une fois, une magnifique image, dans un livre sur la Forêt Vierge qui s'appelait "Histoires Vécues” » (de Saint-Exupéry, 1946)1.

Tl est assez rare, hors du cadre de documents du type « rapport final », que L l'on soit amené à prendre le temps de jeter librement un regard rétrospectif sur un programme de recherche achevé. C'est l'objet de ce texte, placé sous la tutelle du Petit Prince d'Antoine de Saint-Exupéry, personnage qui au fond ne fait pas autre chose : regarder en arrière, vers un lieu quitté et un temps passé, vers une toute petite planète où il vivait comme dans une bulle ou, comme sa rose, sous un globe protecteur ${ }^{2}$. C'est à cette bulle temporelle que je souhaite comparer ici le programme Jugurta ( Justice Spatiale, Gouvernance et Territorialisation dans les villes des Suds»).

1 Toutes les exergues de parties de cet article et plusieurs citations dans le texte sont extraites de ce même ouvrage, je ne le signale donc ici qu'une fois pour toutes.

2 Le choix de mobiliser ici cette œuvre littéraire mérite sans doute explication sinon légitimation. La plus générale, mais qui me semble juste, est que la limite entre littérature et sciences humaines et sociales mérite d'être questionnée (GervaisLambony, 2007) et, plus simplement encore, que bien des géographes mobilisent la littérature dans leurs travaux (ce que, personnellement, je me sens de moins en moins capable de ne pas faire). Mais plus précisément, Le Petit Prince me semble contenir bien des pistes de réflexion sur des questions de méthodologie de recherche (et probablement tout particulièrement en géographie). Par exemple, comme le Petit Prince dont Saint Exupéry nous raconte le voyage de planète en planète ( 7 au total, la dernière qu'il visite étant la Terre), nous avons, au cours des travaux du groupe Jugurta, circulé de ville en ville (et, comme lui, interrogé les habitants) : c'est la base même du comparatisme, dimension essentielle de notre programme. Sur les représentations du juste et de l'injuste, ou plutôt sur la relativité du juste, Saint-Exupéry dit aussi beaucoup dans ce seul livre (dont il ne faut pas oublier qu'il est publié en France en 1946 et écrit durant la Seconde Guerre mondiale), et le personnage du Petit Prince est réellement animé sur le sujet par une capacité de questionnement qui est certainement aussi un modèle méthodologique : interroger et interroger encore, jusqu'à obtenir une réponse (au risque d'agacer le personnage de l'aviateur : " le petit prince ne renonçait jamais à une question, une fois qu'il l'avait posée "). Le Petit Prince enfin, met en cause notre mode même d'appréhension et de compréhension du monde, il n'hésite jamais à questionner les idées reçues, celles des " grandes personnes " qui " se voient importantes comme des baobabs " et que le chercheur court toujours le risque de devenir. On pourrait multiplier les exemples de grande utilité de ce livre, on filera donc dans le présent article cette métaphore du Petit Prince. Ce n'est ni par coquetterie ni parce que c'est en Afrique qu'a lieu la rencontre de l'aviateur avec lui, mais pour des raisons de fond. 
Pour ce faire, on sera amené dans les pages qui suivent à aborder différents niveaux de réflexion. Sur la recherche en sciences humaines et sociales aujourd'hui, sur les actions visant à développer une approche nouvelle, sur la réflexion théorique relative aux dimensions spatiales du social en général, sur l'urbain contemporain, sur les pays dits « du Sud » et la circulation des idées et concepts du Nord au Sud et retour. L'objet du présent texte n'est donc pas de faire une synthèse du programme Jugurta ${ }^{3}$, ni de vanter la qualité des résultats obtenus (et encore moins leur quantité), mais de proposer un regard réflexif sur le programme et une certaine manière de faire de la recherche, qui l'a caractérisé durant le temps qui lui avait été imparti (2007-2012).

Une trentaine d'enseignants-chercheurs ou chercheurs ont participé au programme Jugurta. Ils étaient volontairement divers tant par leurs terrains que par leurs thématiques de recherche habituelles (certains travaillant plutôt sur des questions de gestion et de politique urbaines, d'autres plutôt sur les pratiques et identifications citadines), leur pays d'origine (France, Kenya, Togo, Maroc, Afrique du Sud) et leur âge (des chercheurs dits « seniors » aux doctorants). Tous se sont reconnus dans un objectif commun : montrer, en s'appuyant sur les divers bagages disciplinaires mais aussi sur des travaux de philosophie politique, que la compréhension des interactions entre espace et société est essentielle à celle des injustices sociales. Le concept de justice spatiale, c'est-à-dire l'approche spatiale de la justice sociale entendue dans ses différentes dimensions, tant de distribution équitable que de « reconnaissance », était en effet, au cour de la réflexion de ce groupe de chercheurs.

Le nom, presque acronyme, du programme avait été retenu pour rappeler un autre objectif scientifique : la «distribution » plus « équitable » de la recherche urbaine entre les Suds et les Nords ; il s'agissait en effet, d'adopter un point de vue sur les questions urbaines depuis les Suds, en particulier depuis le continent africain. Jugurtha, roi vaincu de Numidie, mort en prison à Rome, barbare en Occident mais héros en Afrique, convenait fort bien comme figure tutélaire. Malgré une lettre manquante, le nom de notre collectif de recherche n'était donc pas anodin. Peut-être ce désir partagé

3 Le lecteur pourra trouver cette synthèse, mais aussi les nombreuses études empiriques, que la place manque pour développer ici, dans l'ouvrage publié par les membres du groupe Jugurta (Gervais-Lambony et al., 2014). 
de se donner le plaisir de jouer sur les mots tout en décentrant le regard était-il aussi lié au fait que le déroulé de la recherche se faisait dans une période tendue pour l'université et la recherche en France, notre «petite planète » étant alors, comme encore aujourd'hui (dans une continuité qui peut surprendre et provoquer, encore une fois, une certaine déception visà-vis des changements politiques), marquée par des évolutions structurelles fortes, des ruptures temporelles et des débats sur les conditions d'exercice de notre métier. Dans un tel contexte, un programme de recherche financé se présente comme une opportunité, limitée dans le temps et rythmée par la production de points d'étape et de moments partagés, de travailler collectivement et en quelque sorte parallèlement à la «vraie vie ». Ceci pose de réelles questions sur les temporalités de l'activité de recherche, et si je choisis ici d'appeler le programme Jugurta une «bulle », c'est parce que c'est peut-être ainsi que nous avons vécu cette période : une parenthèse au cour du temps ordinaire, une bulle dont je voudrais dans le présent texte explorer et décrire les conditions de constitution d'abord, le mode de fonctionnement interne ensuite et, enfin, les voies pour en prolonger l'existence sous d'autres formes.

\section{Donner du temps, diversifier les temporalités}

«C'était raisonnable autrefois. J'éteignais le matin et j'allumais le soir. [...]

- Et, depuis cette époque, la consigne a changé ?

- La consigne n'a pas changé, dit l'allumeur. C'est bien là le drame ! La planète d'année en année a tourné de plus en plus vite, et la consigne n’a pas changé ! »

Au point de départ d'une réflexion à long terme sur le concept de justice spatiale est une réunion d'unité de recherche à l'Université de Paris Ouest Nanterre La Défense (équipe Gecko, EA 375) lors de laquelle je souhaitais proposer une thématique fédératrice. La part la plus difficile du travail de direction d'une équipe n'est probablement pas tant de parvenir à assurer une gestion administrative que de faire en sorte qu'un véritable échange scientifique s'engage au sein de l'unité, chose d'ailleurs souvent de plus en plus difficile au fur et à mesure que les dimensions des unités 
de recherche s'accroissent sous la pression de modes d'évaluation dominés par la mesure quantitative (répétons la phrase très juste d'Antoine de SaintExupéry : « les grandes personnes aiment les chiffres »). L'échange scientifique suppose le partage d'interrogations communes, interrogations qui doivent être jugées suffisamment importantes pour que les uns et les autres acceptent d'y consacrer du temps, voire d'y « perdre » du temps. Or la question du temps de la recherche est aujourd'hui centrale sous l'effet de pressions qui, comme l'expliquerait l'allumeur de réverbères rencontré par le Petit Prince si on l'interrogeait ${ }^{4}$, relèvent d'une « compression de l'espacetemps » (Harvey, 1990) qui a gagné le monde académique : pression du côté « formation » du métier d'enseignant-chercheur (au rythme accéléré des réformes de l'enseignement supérieur et de la mise en compétition des établissements) ; pression aussi sur le versant « recherche » du métier (processus lourds d'évaluation, individualisation des dossiers combinée à la nécessité du travail collectif, multiplication des appels à projets de plus en plus complexes).

Initiée donc au sein d'une équipe d'accueil universitaire, la réflexion sur la thématique de la justice spatiale a été jugée assez importante pour que l'on y consacre du temps dans des cercles plus larges. Ceci a donné lieu à un faisceau d'actions scientifiques dans lequel s'est inscrit le montage du projet Jugurta en 2007. De ce faisceau, on peut signaler un certain nombre de publications venues nourrir la problématique (Gervais-Lambony, Dufaux, 2009; Bret et al., 2010 ; Blanchon et al., 2011 ; Fol et al., 2013 ; Dufaux, Philifert, 2013 ; Gervais-Lambony et al., 2014 ; Le Blanc et al., 2014). Jugurta n'est donc pas une initiative isolée, mais bien la pièce centrale d'un puzzle complexe qui associe des chercheurs et enseignants-chercheurs bien au-delà des seuls participants au programme. Il convient aussi de replacer cet ensemble dans une temporalité plus longue que celle du seul programme : le commencement lui est antérieur, sa pérennisation est l'objectif. Et ceci a un véritable sens dans la période actuelle : il me

4 La planète de l'allumeur de réverbères est la plus petite de celles visitées par le Petit Prince, l'allumeur y vit seul et son rôle est d'allumer le réverbère au coucher du soleil et de l'éteindre à son lever. Comme sa planète tourne de plus en plus vite, il doit allumer et éteindre de plus en plus souvent, jusqu'au clignotement presque. Sur notre planète scientifique, l'accélération du temps n'est peut-être pas moins sensible. Reste à voir si, par contre, la consigne, pour nous, n'a pas aussi changé. 
semble essentiel de parvenir à inverser une tendance qui, sous l'effet de la contractualisation, raccourcit les temporalités de la recherche. C'est-à-dire que, pour donner du temps à la recherche, on peut suggérer de construire sur le temps long des collectifs scientifiques qui, par périodes, s'inscrivent dans le cadre temporel limité de programmes de recherche de natures diverses. À savoir : inscrire les temporalités brèves de la recherche sur projet dans la temporalité longue de la réflexion scientifique. Pour sortir de la précipitation constante, donc, cela revient à simplement produire des bulles ou parenthèses au sein d'une temporalité plus longue.

Le programme Jugurta a ainsi été une des applications, parmi d'autres, d'une réflexion théorique générale. Les terrains de cette application étaient des villes des Suds, surtout africaines. Il a d'abord fallu se donner le temps de la confrontation des recherches récentes sur ces terrains, et ceci a permis de dégager une constatation commune : une gouvernance urbaine marquée par deux principales évolutions qui mettent toutes deux en jeu des processus de production de territoires. D'une part, la mise en compétition des villes à l'échelle globale pousse les différents acteurs (pouvoirs publics mais aussi acteurs du monde économique) à multiplier les partenariats public-privé qui reposent sur la création de périmètres d'action spécifique, c'est-à-dire que l'on constate une forte territorialisation des politiques publiques censée être mieux à même de répondre à l'hétérogénéité des espaces et des groupes sociaux urbains. D'autre part, se multiplient aussi les productions de territoires locaux portées par des collectifs citadins pour souligner l'inefficacité de la gestion urbaine par les pouvoirs publics et exprimer leurs revendications à l'échelle locale. Et ceci concerne les groupes sociaux urbains dans toute leur diversité : les plus aisés dans des espaces clos sécurisés (sur le modèle des gated communities, nous rencontrions ce type d'espace dans toutes les villes étudiées), les plus pauvres dans des espaces d'exclusion où la territorialisation est un outil de mobilisation. Dans ce second cas, on peut d'ailleurs distinguer les mobilisations qui visent à défendre ou revendiquer des « droits » (droits d'exister dans le cas des quartiers " informels », droit à l'accès aux services urbains, à l'éducation, aux ressources, etc.) et celles qui visent plutôt à maintenir des positions de pouvoir locales, parfois non légales. 
Nous observions donc deux familles différentes de territorialisation, toutes deux sous-tendues par des discours sur leur efficacité (gestionnaire ou mobilisatrice) en même temps que par le souci d'une plus grande « justice ». Notre interrogation de départ a trouvé sa source dans ce constat : territorialisation(s) et justice spatiale sont-elles compatibles ? L'objet du programme Jugurta était de répondre à cette question. Ceci n'était possible qu'en prenant certaines positions par rapport aux débats que l'on peut dire « en amont » sur les définitions des termes utilisés.

\section{Définir}

\subsection{Territoire et territorialisations}

« Mais tous ces travaux familiers lui parurent, ce matin-là, extrêmement doux. Et quand il arrosa une dernière fois la fleur, et se prépara à la mettre à l'abri sous son globe, il se découvrit l'envie de pleurer. »

La planète du Petit Prince est toute petite, vécue comme unique, et il ne prend conscience de son importance pour lui qu'au moment où il la quitte. À la fin, c'est la nostalgie pour cette planète d'origine qui l'emportera : malgré sa peur du serpent et sa peur de la mort, le Petit Prince retourne chez lui. Saint-Exupéry ne nous donne-t-il pas ainsi le modèle même du territoire humain? C'en est en tout cas une des approches possibles, retenue par de nombreux géographes (Di Méo, 1998 ; Gervais-Lambony, 2003) et qui repose sur l'idée du lien entre construction identitaire et territoire. L'originalité du programme Jugurta a été de faire le choix de ne pas faire de choix entre cette famille-là de définition du territoire et celle, plus politique, qui s'inscrit dans une pensée de la relation entre pouvoir et espace, dans la lignée des travaux de Claude Raffestin (1980), mais aussi des auteurs anglophones, pour lesquels le territoire met en jeu avant tout des relations de pouvoir. Il nous a semblé d'emblée essentiel de penser simultanément ces deux logiques, précisément pour comprendre leurs interactions. En conséquence, nous avions à nous appuyer sur deux corpus de travaux scientifiques, d'une part ceux qui étudient les démarcations territoriales établies par des pouvoirs (territorialisation des politiques publiques, manipulations territoriales électorales ou stratégies des entreprises) ; d'autre part ceux qui 
portent sur l'identification d'un territoire local par des groupes sociaux afin de porter sur une scène publique leurs revendications.

Dans tous nos cas d'étude, mais selon des modalités très différentes, la territorialisation des politiques publiques penche dans le sens d'une adaptation à des contextes locaux hétérogènes définis comme territoires, avec leurs modes de gouvernance (formels ou informels) spécifiques, impliquant des formes de participation de la «société civile » locale. La rhétorique de la démocratie participative est souvent utilisée pour justifier ces politiques spatialement différenciées : il s'agit pour les pouvoirs publics soit de renforcer des identités locales préexistantes, soit d'en construire de nouvelles, pour gagner en légitimité. Cette territorialisation s'opère dans un cadre néolibéral, plus ou moins structurant et plus ou moins dominant selon les contextes. L'objectif de croissance économique nécessite de définir des territoires de la croissance et de l'investissement dotés d'une gestion spécifique. Il implique a contrario la relégation des problèmes sociaux dans des espaces dont il faut s'assurer qu'ils ne débordent pas sur les territoires de la croissance (Bénit, Gervais-Lambony, 2003). Les City Improvement Districts associant acteurs publics et privés, étudiés dans le cadre du programme au Cap par Marianne Morange et Alain Dubresson (Gervais-Lambony et al., 2014), pour permettre la mise en place de véritables territoires d'exception, en sont un exemple remarquable, mais on a pu en observer de similaires à Tanger, à Nairobi ou à Lomé. Cependant, d'une territorialisation orchestrée « par le haut », l'on peut facilement glisser vers une demande de transfert de pouvoirs venue du «bas », réclamant au nom de la justice l'application à leur propre territoire du statut d'exception. L'affichage d'une collection de territoires, dans lesquels les pouvoirs publics ont mis en place un mode de gestion différencié, rend possible cette forme de contestation par des groupes sociaux citadins territorialisés ou par des acteurs instrumentalisant les identifications territoriales. C'est précisément du fait de ces interactions, et de ces effets-retours, qu'il nous a fallu jouer sans cesse sur les deux catégories de définition du terme «territoire». 


\subsection{Justice et injustices}

«Je te fais ministre!

- Ministre de quoi?

- De... de la justice!

- Mais il n’y a personne à juger ! »

Étudier la justice spatiale, ce n'est pas juger selon des critères moraux telle organisation spatiale ou telle politique, c'est analyser les conséquences de l'organisation de l'espace en termes de justice et d'injustices sociales, en même temps que les conséquences des injustices sociales sur l'organisation de l'espace ; c'est donc réfléchir aux interactions entre justice et injustices sociales et espace. Quel que soit le type d'espace auquel on applique ce concept, on est toujours conduit à placer au premier plan le politique et les politiques. Que serait une politique territoriale « juste »? Devrait-elle traiter de façon identique tous les espaces ou mettre en œuvre des dispositifs de régulation différenciés pour réduire les injustices là où elles se trouvent? On voit la relation avec le questionnement sur les territorialisations en même temps que l'on dévoile le deuxième problème de définition : qu'est-ce que la justice sociale (et donc spatiale) ? Les débats sur ce sujet s'articulent autour de deux grandes oppositions ou distinctions :

- opposition entre une justice conçue comme "structurelle » (ou "distributive ») et visant à atténuer les inégalités sociospatiales par des mesures politiques et techniques, et une justice pensée comme " procédurale » selon laquelle ce sont les modalités de prise de décision qui conditionnent le caractère juste d'une action ;

- opposition entre des définitions qui se veulent « universalistes », qui pourraient de ce fait être appliquées partout, et celles qui rejettent la possibilité même d'une définition universelle.

La possibilité d'une définition « universelle » du juste et de l'injuste, et donc la question des représentations locales du juste et de l'injuste, s'est rapidement révélée centrale. Dès les premières enquêtes de terrain, il est en effet, apparu que nos définitions de la justice sociale étaient souvent incomprises par les citadins et, donc, inadaptées. Le cas de Maputo a sans doute été le principal révélateur : Karine Ginisty (2014) et Jeanne Vivet (2013) nous rendaient compte de leurs difficultés et étonnements sur le ter- 
rain : le contraste entre ce que nous pouvions définir de notre point de vue comme des injustices (inégalités d'accès aux services urbains, déguerpissements forcés de quartiers de déplacés de la guerre civile pour construire des résidences sécurisées de luxe) et l'absence non seulement de mobilisation collective mais même d'expression de sentiments d'injustice de la part des citadins était flagrant. Certes le contexte politique post-marxiste (et donc une relation spécifique à l'État) était un des éléments qui expliquait la nonmobilisation citadine, mais les politiques publiques locales ou conduites sous la houlette des institutions internationales s'articulaient pourtant sur un discours et des conceptions classiques du juste et de l'injuste. Cette difficulté est bien sûr centrale dans toute démarche qui consiste à tester un modèle théorique mais aussi à mobiliser un même concept sur des terrains différents : une définition du juste ou de l'injuste importée par le chercheur ne pouvait convenir ou en tous cas ne pouvait suffire. Et, audelà, il est aussi apparu que les différents acteurs et les différents groupes sociaux et spatiaux citadins, dans une même ville, fonctionnaient également sur des conceptions du juste différentes voire contradictoires, ce qui était souvent un facteur explicatif de conflits politiques et territoriaux. Le cas des villes sud-africaines, et tout particulièrement de celles de la région de Johannesburg, le démontrait très clairement, ne serait-ce que parce que la diversité des groupes sociaux y est à la fois extrême et marquée historiquement et spatialement. Par exemple, la construction d'un ensemble de logements sociaux à Ekurhuleni, décrite comme juste par les autorités métropolitaines, était dite injuste par les habitants blancs d'un quartier voisin qui considéraient n'avoir pas été consultés, mais aussi par les habitants du bidonville qu'il était prévu de reloger dans ces logements neufs mais qui refusaient un déplacement qui les éloignait de leurs lieux d'emploi : conceptions distributive et procédurale de la justice étaient ici en opposition et portées chacune par des acteurs en conflit. Ceci nous a conduits à travailler en profondeur sur les représentations citadines locales du juste et de l'injuste, puis sur leurs modalités d'expression. Ce fut le premier « glissement de terrain 5 » (au sens de provoqué par la recherche sur le terrain) de notre problématique d'ensemble.

5 Je reprends cette expression des titres des ateliers que l'équipe du laboratoire Gecko avait organisés lors des journées d'études du RTP (Réseau Thématique Prioritaire) Afrique en 2010. Les enregistrements de ces ateliers sont disponibles à l'adresse : http://etudes-africaines.cnrs.fr 
Mais, au point de départ, notre attention était surtout sollicitée par les relations entre les dimensions structurelles et procédurales de la justice spatiale. De ce point de vue, la référence la plus classique est John Rawls (1971). La définition rawlsienne de la justice comme équité est procédurale, parce que l'on juge le caractère juste de telle ou telle décision en fonction de la procédure qui a été suivie pour la prendre en même temps qu'en fonction de son résultat qui se doit d'être le plus favorable possible aux plus faibles. Selon Rawls, c'est en effet le seul objectif qui peut être raisonnablement accepté comme juste par des individus qui seraient placés dans l'ignorance de leur position dans la société. Bien que se distinguant des approches marxistes, jusque-là dominantes et tournées au contraire vers les dimensions structurelles de la justice sociale et soulignant le caractère par essence producteur d'injustices du système capitaliste, John Rawls a été critiqué par un courant de pensée qui se revendiquait plus radicalement procédurale. Iris Marion Young (1990), principale inspiratrice de ce courant, propose de chercher à identifier les injustices dont sont (ou s'estiment) victimes certains groupes, pour poser les bases d'une «théorie de l'injustice ». S'écartant d'une approche centrée sur les inégalités socio-économiques, elle considère que la justice sociale inclut la reconnaissance et l'acceptation de l'altérité, et prône une politique territoriale attentive aux droits des groupes. Pour Young, c'est de la négociation entre groupes sociaux (et non entre individus) que surgit la décision « juste », et non pas d'un idéal abstrait de procédure, comme chez Rawls. Il ne peut donc être question de principes universels.

Sur la base de ces diverses références théoriques, des géographes anglophones (Harvey, 1973 ; Merrifield et Swyngedouw, 1997 ; Soja, 2010 ; Smith, 2000), mais aussi francophones (Reynaud, 1981 ; Bret et al., 2010), se sont penchés sur la notion de justice spatiale; d'autres auteurs ont construit une problématique originale autour de la notion de «ville juste » (Fainstein, 2009 ; Marcuse et al., 2009), c'est-à-dire combinant trois impératifs : démocratie, égalité, reconnaissance. Notre groupe Jugurta s'est inscrit directement dans ces questionnements, en association étroite avec l'équipe qui anime, depuis 2009, la revue en ligne Justice Spatiale / Spatial Justice ${ }^{6}$. Ce collectif plus large, né à l'issue du colloque «Justice et injustice spa-

6 Consultable à l'adresse http://www.jssj.org 
tiales » organisé à l'Université de Nanterre en mars 2008, soutenu par l'UMR LAVUE, rassemble des chercheurs et enseignants-chercheurs appartenant à diverses institutions et unités de recherche, françaises et étrangères, travaillant sur des pays des Nords comme des Suds, unis par un engagement commun dans la réflexion sur les relations entre espace et justice sociale. Ce sur quoi s'accordent ces chercheurs est l'importance des deux dimensions de la justice spatiale. Dimension structurelle d'une part, définie par l'accès égal de tous les citadins aux ressources urbaines, qu'il passe par l'équipement en services des quartiers défavorisés ou par le développement de transports adaptés pour l'accès des quartiers périphériques aux équipements urbains centraux, par exemple ; dimension procédurale d'autre part, qui impose sa négociation entre les acteurs en présence. Toutefois, ce type d'approche soulève la question des articulations ou des possibles contradictions entre justice structurelle et procédurale, ceci d'autant plus que le discours sur la démocratie participative est aisément récupéré par le discours néolibéral : retrait de l'État et ouverture à la multiplicité des acteurs sociaux, acceptation et même renforcement des exceptions territoriales. Ce questionnement sur les interactions entre types de justice sociale se retrouve dans les travaux de Nancy Fraser (2005) qui démontre que toutes les injustices doivent être considérées comme liées à la fois à des questions de redistribution et de reconnaissance et que, selon les cas, c'est en agissant plutôt sur le manque de redistribution ou plutôt sur le manque de reconnaissance que l'on peut les réduire.

Là encore le choix du groupe Jugurta a donc été de mobiliser plusieurs définitions à des fins heuristiques. Cette démarche s'est appuyée sur des méthodologies qui reposaient systématiquement sur des confrontations et comparaisons de terrains différents.

\subsection{Comparer}

« Je me croyais riche d'une fleur unique, et je ne possède qu'une rose ordinaire. »

Le choix d'une approche comparée était une nécessité en même temps qu'un choix assumé. En effet, onze villes avaient été sélectionnées en fonction des terrains des membres du programme : deux villes marocaines 
(Rabat et Tanger), trois villes sud-africaines (Le Cap, Johannesburg, Ekhurhuleni), deux villes kenyanes (Nairobi et Kisumu), la capitale mozambicaine (Maputo), deux villes de la côte du golfe de Guinée (Lomé et Cotonou), une ville secondaire du Nicaragua (Leòn-Sutiaba) et, enfin, les villes d'Équateur. Entre toutes ces villes, comme entre autant de planètes visitées par notre Petit Prince ${ }^{7}$, les différences étaient nombreuses et souvent radicales : de taille, entre les villes multimillionaires et les villes secondaires; de statut, entre les villes capitales nationales et les autres; de poids économique et donc de ressources des pouvoirs nationaux et locaux ; de contexte historique évidemment, de contexte politique national aussi. Confrontés, par choix, à cette diversité, notre méthode a consisté à travailler sur chaque terrain dans la même perspective d'analyse des processus de territorialisation (processus eux-mêmes divers, comme indiqué précédemment : territorialisations par les politiques publiques, territorialisation par les individus et groupes sociaux) et de leurs liens avec la notion de justice spatiale. Cette démarche imposait à chaque chercheur de reconsidérer son propre terrain (sa propre rose, unique et apprivoisée ${ }^{8}$ ) à la lumière d'une problématique nouvelle, et, à chaque fois que cela a été possible, des croisements de terrains ont été réalisés. Pour qu'une telle démarche soit possible il fallait adopter une grille de lecture commune ; nous l'avions initialement organisée selon les questions de recherche suivantes :

- Quelles sont les politiques territorialisées/spatialisées mises en œuvre?

- Quelle est la dimension « participative » de ces politiques ? Dans quelles mesures et comment jouent-elles sur les identités locales? Les produc-

7 II me semble assez clair que le Petit Prince réalise l'exercice géographique comparatif par excellence. Partant de chez lui, circulant d'un lieu à l'autre et le confrontant au sien (et en étant conscient), posant avec insistance des questions, dégageant ainsi le singulier du général, se servant d'un cas pour en mieux comprendre un autre, difficile de contester qu'ainsi il enrichit sa compréhension du monde.

8 Sur la planète du Petit Prince, il n'y a qu'une seule fleur, sa rose qu'il croit donc unique. C'est sur la Terre qu'il arrive dans un jardin "fleuri de roses " et c'est alors qu'il pense, tristement, " je me croyais riche d'une fleur unique, et je ne possède qu'une rose ordinaire ". Ce n'est que plus tard qu'il comprendra que sa rose est unique parce qu'il a tissé avec elle une relation unique, il l'a apprivoisée (c'est ce que lui apprend le renard qu'il rencontre ensuite). La relation du chercheur à son terrain me semble être d'une nature assez proche et, du coup, le rôle de la comparaison essentiel : tout terrain, en l'occurrence toute ville, n'est qu'un parmi d'autres et on risque toujours de l'oublier, mais tout terrain de recherche est pourtant bien unique car il est d'abord une relation entre le chercheur et lui, une construction. 
tions identitaires, en retour, sont-elles instrumentalisées pour appuyer des revendications citadines d'accès au pouvoir et aux ressources?

- Existe-t-il une régulation d'ensemble ou assiste-t-on à la juxtaposition de politiques non coordonnées entre elles?

- L'espace en tant que tel est-il un simple cadre ou bien est-il au cœur de la production de la justice ou de l'injustice?

Nos terrains de recherche présentaient sur tous ces points plus de divergences que de convergences, et c'est bien ce que nous recherchions. Nous avions établi pour commencer une grille de lecture simple qui s'appuyait sur deux oppositions principales : d'une part, les villes où un pouvoir politique fort et doté de moyens financiers puissants conduisait une politique de territorialisation à celles où ce n'était pas le cas ; d'autre part, les villes ou l'idée de justice, redistributive ou procédurale, était un moteur des politiques aux autres. Par exemple, à Nairobi, pas de forte territorialisation par le haut, ni de nette politique de justice sociale, contrairement aux villes sud-africaines et même marocaines. La ville de Lomé est, de ce point vue, proche de la capitale kenyane : absence plus nette encore de démocratie ; faiblesse, voire absence, du pouvoir municipal ; discordance entre le territoire de la municipalité et celui de l'agglomération; quasi-absence à l'échelle de la ville d'une volonté politique de redistribution ; importance des « arrangements » dans la gestion urbaine. Les villes sud-africaines (Le Cap, Johannesburg et Ekurhuleni) se caractérisent au contraire par une recherche affichée d'un équilibre entre redistribution (réparatrice des inégalités structurelles héritées de l'apartheid) et participation (dans la lignée des mouvements civiques locaux de libération). Les villes du Maroc concentrent également l'attention des pouvoirs publics, à la fois soucieux de placer leur pays dans le jeu de la concurrence mondiale et conscients de la multiplicité des problèmes qui s'expriment à l'échelle locale. Maputo présente à l'évidence un cas de figure différent qui est caractéristique du passage d'un régime marxiste-léniniste officiel à un régime néolibéral. Enfin, le cas des villes d'Équateur et l'étude du couple formé par le quartier indigène de Sutiaba et la ville " espagnole » de León (dont la séparation est le résultat d'un long processus historique de séparation des groupes ethniques depuis l'époque coloniale) ont pu servir de référents comparatifs parce qu'elles étaient les seules villes non africaines de notre échantillon. 
Nous étions satisfaits, lors des premiers séminaires de notre groupe, organisés à Nanterre puis à Johannesburg, d'être parvenus à établir cette grille commune pour interroger nos terrains respectifs. Mais nous allions alors rencontrer notre deuxième « glissement de terrain » et être amenés à décider que cette grille n'était pas opératoire et que différences et similitudes entre nos « roses » étaient plus complexes à comprendre qu'il ne nous avait d'abord semblé.

\subsection{Glissements de terrain, encore}

« Le géographe est trop important pour flâner. Il ne quitte pas son bureau. Mais il y reçoit des explorateurs. Il les interroge, et il prend en note leurs souvenirs. »

La sixième planète que le Petit Prince visite lors de son voyage vers la Terre est habitée par un géographe, « un vieux monsieur qui écrivait d'énormes livres », lequel lui explique sa complémentarité avec les explorateurs. Ce sont ces derniers qui vont sur le terrain et lui rapportent des informations. Et peut-être est-il en effet, question de complémentarité, ou bien d'une double face du géographe ? L'un des liens entre les participants au programme Jugurta était en tous cas la volonté, à la différence du géographe rencontré par le Petit Prince, de faire du terrain dans le cadre même du programme, en même temps qu'ils s'engageaient dans une réflexion théorique à partir de situations déjà explorées. Il s'agissait aussi de rendre possible les réorientations scientifiques en fonction des résultats de terrain, c'est-à-dire des « glissements (théoriques) de terrain ». On a déjà indiqué le premier de ces glissements : l'intégration dans le programme d'une réflexion sur les représentations locales et du juste et de l'injuste qui, seule, pouvait permettre d'éviter d'appliquer des concepts inadéquats. Ceci était d'autant plus essentiel que bien des travaux sur la justice spatiale viennent d'auteurs états-uniens travaillant sur des villes d'Amérique du Nord. Comment pouvaient-ils être mobilisés ailleurs, et tout particulièrement dans des villes africaines ou latino-américaines, villes où la pauvreté est dans des proportions peu comparables à l'Amérique du Nord, où les États sont aussi le plus souvent bien plus dépourvus de moyens, où les pratiques informelles sont bien plus développées? La question de la spécificité éventuelle des villes des Suds n'était volontairement pas centrale dans 
notre travail ; au contraire, même, nous voulions éviter cette approche pour considérer que l'on avait simplement affaire à des villes, des villes à part entière (Robinson, 2006). Mais la prise en compte des points de vue et des discours des citadins de chacune des villes étudiées s'est révélée essentielle pour éviter les dérives résultant du « placage » d'un modèle. Ainsi, comment poser les questions de la même manière à Lomé, dans le cadre d'un système politique autoritaire où c'est d'abord dans la nature même du pouvoir politique que les citadins voient l'injustice, et en Afrique du Sud où, théoriquement, toute la politique publique est orientée vers la réparation des injustices? Ou bien encore, comment ne pas prendre en compte la particularité du cas kenyan où l'idée de justice est avant tout liée à la question foncière (de l'accaparement des terres, de l'époque coloniale à aujourd'hui, par les élites) ? Et que dire du cas marocain et de la conception du juste dans le système monarchique ? De ces différences découlent des formes de mobilisation différentes, ou l'absence de mobilisation d'ailleurs, et des appréciations éventuellement radicalement opposées de ce qui serait juste ou injuste.

Quoi qu'il en soit, le deuxième glissement est celui qui nous a conduits à approfondir la réflexion sur les échelles.

Tout d'abord, nous avons constaté que réfléchir à l'échelle d'une agglomération dans son ensemble était peu efficace. La catégorisation de nos cas d'étude à cette échelle était peu opérante parce que c'était à l'échelle intra-urbaine que les comparaisons étaient le plus fructueuses : notre grille de lecture et de comparaison initiale ne fonctionnait tout simplement pas. Par exemple, que ce soit à Nairobi, au Cap ou à Rabat, le centre-ville fait l'objet d'une politique publique forte, en partenariat public-privé le plus souvent, donc d'une forte territorialisation par le haut. Mais dans d'autres espaces des mêmes villes, ce type de territorialisation est faible et les formes d’injustice spatiale radicalement différentes.

Plus fondamentalement, l'assimilation fréquente d'un niveau de gouvernement et de prise de décision à une échelle spatiale donnée nous a posé des problèmes théoriques importants. Il a donc fallu clarifier ce point en posant les questions suivantes : comment le découpage de l'espace en niveaux peut-il traduire un rapport de pouvoirs plus ou moins 
juste ? Comment peut-il également orienter le jeu des acteurs vers davantage, ou vers moins, de justice ? Enfin, les compétences des citadins et des groupes sociaux citadins à circuler d'une échelle à l'autre sans se laisser enfermer dans une échelle prétendue locale nous sont apparues comme un angle mort des recherches sur la justice spatiale : dans quelle mesure un cadre scalaire fluide permettant une importante mobilité des acteurs entre les échelles ne peut-il pas être considéré comme plus juste ? Tenter de répondre à ces questions nous conduisait aussi à sortir notre recherche du cadre souvent contraint de l'approche par le local (échelle a priori privilégiée pour la mise en œuvre d'une justice procédurale, par opposition à l'échelle métropolitaine plus propice à la redistribution) ; c'était aussi une condition pour comprendre comment pouvoirs urbains et citadins pouvaient également agir à d'autres échelles ou au moins en tenant compte des autres échelles. L'a priori selon lequel telle ou telle échelle territoriale serait plus favorable à tel ou tel type de justice sociale continuait de trop influencer la réflexion. Ainsi, les territorialisations que l'on a brièvement décrites plus haut se présentent comme des formes de recours au «local » pour résoudre les questions urbaines. Or l'on sait que ces territorialisations, en tous cas dans les villes sur lesquelles nous avons travaillé et plus généralement dans les villes des Suds, ont été concomitantes d'une augmentation des inégalités sociales, d'une diminution des redistributions de ressources et, donc, de reculs sur le plan de la « justice structurelle ». Aujourd'hui, certaines formes de recentralisation à l'échelle métropolitaine (par exemple de manière nette en Afrique du Sud) s'opèrent, mais dans un contexte idéologique nouveau puisqu'il ne s'agit plus seulement de centraliser pour redistribuer, mais aussi de privatiser ou, selon les cas, d'établir des partenariats avec les milieux d'affaires. Dans ce nouveau contexte, l'appel à une plus grande justice procédurale par des mouvements sociaux ou des associations locales de résidents peut être considéré comme une forme de résistance aux dogmes néolibéraux ; mais cela permet-il d'aller au-delà de victoires locales peu aptes à réorienter les politiques à de plus vastes échelles (Purcell, 2006) ? Ainsi, qu'un quartier informel de Lomé parvienne à échapper (d'ailleurs pour un temps seulement) au déplacement forcé alors qu'il est situé sur la zone franche portuaire (forme extrême d'exception territoriale décidée d'« en haut » et vantée comme juste car devant avoir des effets redistributeurs à l'échelle de la ville et du pays) ne modifie 
en rien la politique urbaine, d'ailleurs entièrement contrôlée au niveau central puisque le pouvoir municipal (le maire en l'occurrence et son équipe) est nommé et non élu.

La question de l'échelle de gouvernement est donc centrale, et la manipulation de différentes échelles est un enjeu de pouvoir important (Brenner, 2001) pour tous les acteurs de la gouvernance : les pouvoirs publics, mais aussi les partis politiques et les mouvements sociaux et associations de résidents. Mais que sont les « échelles » dont on parle ? Elles n'existent pas, en soi, mais sont produites par des acteurs sociaux et politiques (Harvey, 200o) dont elles servent les objectifs. En conséquence, ce n'est qu'en fonction de ces objectifs que l'on peut dire l'efficacité plus ou moins grande d'une « échelle » et, partant, il ne saurait être question d'un lien mécanique entre une échelle de gouvernement et son caractère « juste » ou « injuste », de l'échelle locale pas plus que d'une autre. Mais on peut aussi revenir aux écrits d'Iris Marion Young qui est parfaitement claire sur ce point : « decision making structures and processes at the local level often tend to create and exacerbate injustice9 » (Young, 1990, p. 244). D’où le problème de savoir comment sortir du dilemme entre la nécessité d'une « local self-determination $^{10}$ » et celle d'un « region-wide aknowledgement of the legitimate interests of others ${ }^{11} »$ (Young, 200o, p. 230). ? C'est-à-dire comment rendre possible la circulation des acteurs d'une échelle à l'autre.

Il n'en reste pas moins que ce qui est vécu comme territoire local est une des bases des mobilisations citadines pour plus de justice. Et, bien souvent, cette échelle locale est associée à une référence au temps passé, à une perte ou à un ancrage. Cette question de la référence au passé comme constitutive des représentations de la justice spatiale fut certainement notre troisième plus important glissement de terrain, et il est donc lié à la question des échelles. Les habitants des villes sont confrontés au changement, non pas seulement cyclique mais aussi sans retour. C'est une

9 "Les structures et processus de prise de décision à l'échelle locale ont souvent pour effet de créer ou d'exacerber l'injustice " (traduction libre).

10 " Auto-détermination locale" (traduction libre).

11 "Reconnaissance à l'échelle métropolitaine de la légitimité des intérêts des autres » (traduction libre). 
caractéristique de la citadinité que nous connaissons depuis Charles Baudelaire : «Le vieux Paris n'est plus (la forme d'une ville / Change plus vite, hélas! que le cour d'un mortel)». C'est une conséquence du processus de « destruction créatrice » décrit par David Harvey (1990), dont on sait les conséquences sociales (Veschambre, 2008); il en découle un sentiment qui est donc « a historical emotion » (Boym, 2OO1; XVI), la « nostalgie ». La nostalgie me semble être une des composantes de la relation des sociétés urbaines à leurs espaces et de l'expérience citadine en général (Lepetit, 1995). Elle est accentuée par l'accélération des transformations sociales et spatiales, telles que celles qui caractérisent les villes aujourd'hui. Pour les citadins, il s'agit de gérer à la fois les transformations rapides de leur ville, d'intégrer des référents temporels nouveaux, ou encore, dans certains cas, de constater le manque de changement. Les mobilisations actuelles pour plus de « justice » sont aussi de ces différents ordres : refus du changement ou demande de changement, toujours par référence à un passé regretté ou honni. Dans les entretiens de terrain menés dans toutes les villes étudiées dans le cadre du programme Jugurta, il est en effet, apparu qu'un discours sur le juste et l'injuste se tenait presque nécessairement par comparaison. Cette comparaison pouvait se faire avec d'autres lieux (visités dans le cas de citadins ayant un temps migré à l'étranger, ou imaginés) ou avec d'autres temps, des temps passés et perdus. Ces temps perdus sont bien sûr souvent ceux de la jeunesse ou de l'enfance, c'est le seul «véritable paradis » de Proust mais aussi le temps vers lequel le Petit Prince de Saint-Exupéry nous ramène. Ils sont cependant, tout autant que les échelles ou les territoires, des productions sociales. Un ensemble de productions sociales, en fait, et les groupes sociaux comme les pouvoirs mobilisent différentes représentations du passé qui, bien souvent, s'opposent. La récurrence des discours « nostalgiques » est en effet, frappante dans la ville contemporaine, mais ces discours sont de natures très diverses et mobilisés à des fins elles aussi diverses : par le politique pour construire une identification légitimante, par les mouvements sociaux éventuellement territorialisés pour défendre leur droit d'usage de l'espace, par les acteurs économiques pour commercialiser la mémoire d’un passé idéalisé, par les citadins en général enfin, pour produire simplement un discours qui ordonne leur univers sans cesse changeant (Gervais-Lambony, 2012). La prolifération des références au passé est donc bien liée à l'essor des processus de territorialisation (Di Méo, 1998 ; Roncayolo, 20O2) et au fait que les revendications identitaires territoriales 
de « reconnaissance » se multiplient, s'appuyant sur des territoires locaux, qu'elles produisent, et sur des discours sur le passé (mobilisé aussi pour des raisons tactiques). Les mémoires citadines deviennent donc un véritable enjeu politique et c'est ce qui nous a conduits à poser la question du « droit à la mémoire » pour les citadins comme partie intégrante du «droit à la ville » (Lefebvre, 1972).

Référence à un passé idéalisé, production du local et sentiment d'injustice sont liés. Arjun Appadurai offre des clés importantes pour le comprendre. Il propose en effet, de distinguer l'idée de local de celle d'espace et d'échelle : « la localité est avant tout une question de relation et de contexte, plutôt que d'échelle ou d'espace » (Appadurai, 1996, p. 257). Plus exactement, dans le monde globalisé, la localité ne serait plus une question d'espace, par distinction d'avec la localité ethnographique classique dans laquelle «la localisation spatiale, l'interaction quotidienne et l'échelle sociale » (p. 258) coïncidaient. Il y aurait donc un malentendu : nous pensons le local en termes spatiaux alors qu'il est relationnel, il est une "structure de sentiment »; les «techniques de production du local » (nommer, délimiter, marquer...) utilisent l'espace, s'appuient sur l'espace, mais pour fabriquer du sentiment. Est-ce que le retour au local comme échelle « préférée » n'est d'ailleurs pas une tentative de reconstruction nostalgique d'une localité caractérisée par la coïncidence entre localisation spatiale, interaction quotidienne et échelle sociale ? Reconstruction impossible et aussi par définition exclusive et injuste dans les métropoles que nous étudions, reconstruction impossible aussi car elle confond étendue spatiale et groupe social. Dès lors, nous sortons de la question de l'échelle, mais nous pouvons y revenir : si le local est une structure de sentiment et n'a pas (ou plus) de relation directe avec l'échelle ou une certaine échelle, cette structure de sentiment peut être produite à toutes les échelles et donc à l'échelle métropolitaine aussi bien qu'à celle du quartier.

C'est donc bien sur l'importance du « sentiment » qu'il me semble approprié de conclure. 


\section{Une conclusion très courte et très sentimentale}

«[...] je veux bien dédier ce livre à l'enfant qu'a été autrefois cette grande personne. Toutes les grandes personnes ont d'abord été des enfants. (Mais peu d'entre elles s'en souviennent). Je corrige donc ma dédicace : à Léon Werth quand il était un petit garçon. »

Comme Saint-Exupéry en exergue de son Petit Prince, Amartya Sen ouvre la préface de son livre de 2009 sur l'idée de justice par un hommage aux enfants, en ce qu'ils sont dotés d'une capacité au sentiment d'injustice souvent perdue par les adultes. Ce «sentiment d'injustice peut senir de signal de départ et nous mettre en mouvement » (Sen, 2009, p. 12) vers une analyse critique et une réflexion théorique (qui doit aussi intégrer, on l'a vu, une analyse des sentiments ou représentations des citadins). C'est, à mon sens, le processus qui a permis le bon fonctionnement de la bulle Jugurta et, au-delà, l'ampleur prise actuellement par la réflexion sur le concept de justice spatiale, réflexion dont j'espère avoir montré qu'elle ne pouvait être fructueuse que parce que articulée sur des travaux empiriques de terrain. C'est aussi précisément pourquoi il me semblait qu'il fallait que Jugurtha rencontrât un jour le Petit Prince... et pourquoi il faut encore et encore rappeler que les sentiments sont des «choses sérieuses».

Ces « choses sérieuses » le sont tout particulièrement pour la recherche en sciences sociales car il se pourrait bien, finalement, qu'elles en soient le principal moteur. Plus modestement, cela a été le cas du programme Jugurta, il me semble en tous cas. L'exercice difficile ensuite, en cas d'engagement sentimental dans la recherche, est bien sûr de domestiquer le sentiment luimême ou plus exactement sans doute de l'apprivoiser comme le Petit Prince apprivoise le renard et, ce faisant, donne sens à son environnement :

«Je ne mange pas de pain. Le blé pour moi est inutile. Les champs de blé ne me rappellent rien. [...] Mais tu as des cheveux couleur d'or. Alors ce sera merveilleux quand tu m'auras apprivoisé ! Le blé, qui est doré, me fera souvenir de toi. [...]

- Mais tu vas pleurer ! [...] Alors tu n’y gagnes rien!

- J'y gagne [...] à cause de la couleur du blé. » 
On a à y gagner « la couleur du blé », certes, mais aussi l'impression (et peut-être d'ailleurs simplement l'illusion) d'une recherche pas inutile.

\section{AUTEUR}

\section{Philippe Gervais-Lambony}

Professeur de géographie à l'université Paris-Ouest-Nanterre-La Défense et à l'Institut universitaire de France, UMR Lavue (équipe Mosaïques). 


\section{BIBLIOGRAPHIE}

Appadurai A., 2001, Après le colonialisme, (éd. originale 1996), Paris, Payot.

Bénit C., Gervais-Lambony P., 2003, La mondialisation comme instrument politique local dans les métropoles sud-africaines (Johannesburg et Ekhuruleni) : les " pauvres " face aux " vitrines ", Annales de géographie, n 634, pp. 628-645.

Blanchon D., Gardin J., Moreau S., (éd), 2011, Justice et injustices environnementales, Nanterre, Presses universitaires de Paris Ouest.

Boym S., 2001, The Future of Nostalgia, New York, Basic Books.

Brenner N., 2001, The limits to scale? Methodological reflections on scalar structuration, Progress in Human Geography, 25 (4), pp. 591-614.

Bret B., Gervais-Lambony P., Hancock C., Landy F., (éd), 2010, Justice et injustices spatiales, Nanterre, Presses universitaires de Paris Ouest.

Di Méo G., 1998, Géographie sociale et territoires, Paris, Nathan.

Dufaux F., Philifert P., (éd), 2013, Justice spatiale et politiques territoriales, Nanterre, Presses universitaires de Paris Ouest.

Fainstein S., 2009, Spatial Justice and Planning, justice spatiale / spatial justice $n^{\circ}$ 01, http://www.jssj.org/article/justice-spatiale-et-amenagement-urbain/

Fol S., Lehman-Frisch S., Morange M., (éd.), 2013, Ségrégation et Justice spatile, Nanterre, Presses universitaires de Paris Ouest.

Fraser N., 2005, Qu'est-ce que la justice sociale? Reconnaissance et distribution, Paris, La Découverte.
Gervais-Lambony P., 2003, Territoires citadins, 4 villes africaines, Paris, Belin.

Gervais-Lambony P., 2012, Nostalgies citadines en Afrique Sud, Espaces/ Temps.net, http://espacestemps.net/ document9459.html.

Gervais-Lambony P., Bénit-Gbaffou C., Musset A., Piermay J.-L., Planel S., (éd.), 2014, La Justice spatiale et la ville. Regards du Sud, Paris, Karthala.

Gervais-Lambony P., Dufaux F., 2009, Justice... spatiale!, Annales de Géographie, 665-666, pp. 3-16.

Gervais-Lambony P., 2007, Nouvel espace, nouvelle littérature et retour. Écrire les suburbs de Johannesburg. Remarques autour du recueil d'Ivan Vladislavic: The Exploded View, Bulletin de I'Association des Géographes Français, $n^{\circ} 3$, pp. 275-286.

Ginisty K., 2014, Inégalités et (in)justices spatiales à Maputo (Mozambique). Pratiques des services urbains, thèse de doctorat en géographie, Université de Paris Ouest Nanterre La Défense.

Harvey D., 1973, Social Justice and the City, Londres, Edward Arnold.

Harvey D., 1990, The condition of postmodernity, New York, Blackwell.

Harvey D., 2000, Spaces of Hope, Berkeley, University of California Press.

Le Blanc A., Piermay J.-L., GervaisLambony P., Giroud M., Pierdet C., Rufat S., (éd.), 2014, Métropoles en débats, Nanterre, Presses universitaires de Paris Ouest.

Lefebvre H., 1972, Espace et politique, Paris, Anthropos. 
Lepetit B., 1995, Le présent de l'histoire, in Les formes de l'expérience, une autre histoire sociale, Paris, Albin Michel, pp. 273-298.

Marcuse P., Connolly J., Novy J., Olivo I., Potter C., Steil H., (éd.), 2009, Searching for the Just City: Debates in Urban Theory and Practice, Abingdon, Routledge.

Merrifield A., Swyngedouw E., (éd.), 1997, The Urbanization of Injustice, New York University Press.

Purcell M., 2006, Urban democracy and the local trap, Urban Studies, vol. 43, 11, pp. 1921-1941.

Raffestin C., 1980, Pour une géographie du pouvoir, Genève, LITEC.

Rawls J., 1971, A Theory of Justice, Cambridge, Harvard University Press.

Reynaud A., 1981, Société, espace et justice, Paris, Presses Universitaires de France.

Robinson J., 2006, Ordinary Cities. Between modernity and development, New York, Routledge.
Roncayolo M., 2002, Lectures de villes. Formes et temps, Marseille, Éditions Parenthèses.

Saint-Exupéry (de) A., 1946, Le Petit Prince, Paris, Gallimard.

Sen A., 2009, L'idée de justice, Paris, Flammarion.

Smith David M., 2000, Moral Geographies: Ethics in a World of Difference, Edinburgh, Edinburgh University Press.

Soja E. W., 2010, Seeking Spatial Justice, Minneapolis, University of Minnesota Press.

Veschambre V., 2008, Traces et mémoires urbaines. Enjeux sociaux de la patrimonialisation et de la démolition, Rennes, PUR.

Vivet J., 2012, Les déplacés de guerre dans la ville: I'exemple de Maputo, Paris, Éditions Karthala-Ifas.

Young I. M., 1990, Justice and the Politics of Difference, Princeton, Princeton University Press.

Young I. M., 2000, Inclusion and Democracy, Oxford, Oxford University Press. 